\title{
Maximal diameter sphere theorem for manifolds with nonconstant radial curvature *!
}

\author{
Nathaphon BOONNAM
}

\begin{abstract}
We generalize the maximal diameter sphere theorem due to Toponogov by means of the radial curvature. As a corollary to our main theorem, we prove that for a complete connected Riemannian $n$-manifold $M$ having radial sectional curvature at a point bounded from below by the radial curvature function of an ellipsoid of prolate type, the diameter of $M$ does not exceed the diameter of the ellipsoid, and if the diameter of $M$ equals that of the ellipsoid, then $M$ is isometric to the $n$-dimensional ellipsoid of revolution.
\end{abstract}

\section{Introduction}

The maximal diameter sphere theorem due to Toponogov says that for a complete connected Riemannian manifold $M$ whose sectional curvature is bounded from below by a positive constant $H$, the diameter of $M$ does not exceed $\pi / \sqrt{H}$ and if the diameter of $M$ equals $\pi / \sqrt{H}$, then $M$ is isometric to the sphere with radius $\sqrt{H}$. This theorem was generalized by Cheng [Ch] for a complete connected Riemannian manifold whose Ricci curvature is bounded from below by a positive constant $H$.

A natural extension of the maximal diameter sphere theorem by the radial curvature would be:

For a complete connected Riemannian manifold $M$ whose radial sectional curvature at a point $p$ is not less than a positive constant $H$,

(A) is the diameter of $M$ at most $\pi / \sqrt{H}$ ?

(B) Furthermore, if the diameter of $M$ equals $\pi / \sqrt{H}$, is $M$ isometric to the sphere with the radius $\sqrt{H}$ ?

Notice that the problem (A) can be affirmatively solved. It is an easy consequence from Theorem 2.2 (or the Main theorem in [SST]). Here, we define the radial plane and radial curvature from a point $p$ of a complete connected Riemannian manifold $M$. For each point $q$ distinct from the point $p$, a 2-dimensional linear subspace $\sigma$ of $T_{q} M$ is called a

\footnotetext{
* Mathematics Subject Classification (2010) : 53C22.

${ }^{\dagger}$ Keywords: maximal diameter sphere theorem, 2-sphere of revolution, ellipsoid, Toponogov comparison theorem
} 
radial plane at $q$ if there exists a unit speed minimal geodesic segment $\gamma:[0, d(p, q)] \rightarrow M$ satisfying $\sigma \ni \gamma^{\prime}(d(p, q))$ and the sectional curvature $K(\sigma)$ of $\sigma$ is called a radial curvature at $p$.

The problem (B) is still open, but one can generalize the maximal diameter sphere theorem for a manifold which has radial curvature at a point bounded from below by the radial curvature function of a 2-sphere of revolution, which will be defined later, if the 2 -sphere of revolution belongs to a certain class.

For introducing this class of a 2-sphere of revolution, we start to define a 2-sphere of revolution.

Let $\widetilde{M}$ denote a complete Riemannian manifold homeomorphic to a 2 -sphere. $\widetilde{M}$ is called a 2-sphere of revolution if $\widetilde{M}$ admits a point $\tilde{p}$ such that for any two points $\tilde{q}_{1}, \tilde{q}_{2}$ on $\widetilde{M}$ with $d\left(\tilde{p}, \tilde{q}_{1}\right)=d\left(\tilde{p}, \tilde{q}_{2}\right)$, where $d($,$) denotes the Riemannian distance function, there$ exists an isometry $f$ on $\widetilde{M}$ satisfying $f\left(\tilde{q}_{1}\right)=\tilde{q}_{2}$ and $f(\tilde{p})=\tilde{p}$. The point $\tilde{p}$ is called a pole of $\widetilde{M}$. It is proved in $[\mathrm{ST}$ ] that $\widetilde{M}$ has another pole $\tilde{q}$ and the Riemannian metric $g$ of $\widetilde{M}$ is expressed as $g=d r^{2}+m(r)^{2} d \theta^{2}$ on $\widetilde{M} \backslash\{\tilde{p}, \tilde{q}\}$, where $(r, \theta)$ denote geodesic polar coordinates around $\tilde{p}$ and

$$
m(r(x)):=\sqrt{g\left(\left(\frac{\partial}{\partial \theta}\right)_{x},\left(\frac{\partial}{\partial \theta}\right)_{x}\right)} .
$$

Hence $\widetilde{M}$ has a pair of poles $\tilde{p}$ and $\tilde{q}$. In what follows, $\tilde{p}$ denotes a pole of $\widetilde{M}$ and we fix it. Each unit speed geodesic emanating from $\tilde{p}$ is called a meridian. It is observed in [ST] that each meridian $\mu:[0,4 a] \rightarrow \widetilde{M}$, where $a:=\frac{1}{2} d(\tilde{p}, \tilde{q})$, passes through $\tilde{q}$ and is periodic, hence, $\mu(0)=\mu(4 a)=\tilde{p}, \mu^{\prime}(0)=\mu^{\prime}(4 a)$. The function $G \circ \mu:[0,2 a] \rightarrow R$ is called the radial curvature function of $\widetilde{M}$, where $G$ denotes the Gaussian curvature of $\widetilde{M}$.

A 2-sphere of revolution $\widetilde{M}$ with a pair of poles $\tilde{p}$ and $\tilde{q}$ is called a model surface if $\widetilde{M}$ satisfies the following two properties:

(1.1) $\widetilde{M}$ has a reflective symmetry with respect to the equator, $r=a=\frac{1}{2} d(\tilde{p}, \tilde{q})$.

(1.2) The Gaussian curvature $G$ of $\widetilde{M}$ is strictly decreasing along a meridian from the point $\tilde{p}$ to the point on the equator.

A typical example of a model surface is an ellipsoid of prolate type, i.e., the surface defined by

$$
\frac{x^{2}+y^{2}}{a^{2}}+\frac{z^{2}}{b^{2}}=1, \quad b>a>0 .
$$

The points $(0,0, \pm b)$ are a pair of poles and $z=0$ is the equator.

The Gaussian curvature of a model surface is not always positive everywhere. In [ST], an interesting model surface was introduced. The surface generated by the $(x, z)$-plane curve $(m(t), 0, z(t))$ is a model surface, where

$$
m(t):=\frac{\sqrt{3}}{10}\left(9 \sin \frac{\sqrt{3}}{9} t+7 \sin \frac{\sqrt{3}}{3} t\right), \quad z(t):=\int_{0}^{t} \sqrt{1-m^{\prime}(t)^{2}} d t .
$$

It is easy to see that the Gaussian curvature of the equator $r=3 \sqrt{3} \pi / 2$ is -1 . 
Let $M$ be a complete connected $n$-dimensional Riemannian manifold with a base point $p . M$ is said to have radial sectional curvature at $p$ bounded from below by the radial curvature function $G \circ \mu$ of a model surface $\widetilde{M}$ if for any point $q(\neq p)$ and any radial plane $\sigma \subset T_{q} M$ at $q$, the sectional curvature $K(\sigma)$ of $M$ satisfies $K(\sigma) \geq G \circ \mu(d(p, q))$.

For each 2-dimensional model $\widetilde{M}$ with a Riemannian metric $d r^{2}+m(r)^{2} d \theta^{2}$, we define an $n$-dimensional model $\widetilde{M}^{n}$ homeomorphic to an $n$-sphere $S^{n}$ with a Riemannian metric

$$
g^{*}=d r^{2}+m(r)^{2} d \Theta^{2},
$$

where $d \Theta^{2}$ denotes the Riemannian metric of the $(n-1)$-dimensional unit sphere $S^{n-1}(1)$. For example, the $n$-dimensional model of the ellipsoid above is the $n$-dimensional ellipsoid defined by

$$
\sum_{i=1}^{n} \frac{x_{i}^{2}}{a^{2}}+\frac{x_{n+1}^{2}}{b^{2}}=1 .
$$

In this paper, we generalize the maximal diameter sphere theorem as follows:

Main Theorem Let $M$ be a complete connected n-dimensional Riemannian manifold with a base point $p$. If $M$ has radial sectional curvature at $p$ bounded from below by the radial curvature function of a model surface $\widetilde{M}$, then the diameter of $M$ does not exceed the diameter of $\widetilde{M}$ and furthermore if the diameter of $M$ equals that of $\widetilde{M}$, then $M$ is isometric to the $n$-dimensional model $\widetilde{M}^{n}$.

As a corollary, we get an interesting result:

Corollary to Main Theorem For any complete connected n-dimensional Riemannian manifold $M$ having radial sectional curvature at a point $p$ bounded from below by the radial curvature function of the ellipsoid $\widetilde{M}$ defined by

$$
\frac{x^{2}+y^{2}}{a^{2}}+\frac{z^{2}}{b^{2}}=1, \quad b>a>0
$$

the diameter of $M$ does not exceed the diameter of $\widetilde{M}$ and if the diameter of $M$ equals that of $\widetilde{M}$, then $M$ is isometric to the $n$-dimensional ellipsoid $\sum_{i=1}^{n} \frac{x_{i}^{2}}{a^{2}}+\frac{x_{n+1}^{2}}{b^{2}}=1$.

We refer to [CE] for basic tools in Riemannian Geometry, and [SST] for some properties of geodesics on a surface of revolution.

The present author would like to deeply express thanks to Professor Minoru Tanaka for suggesting the Main Theorem and giving him various comments.

\section{Preliminaries}

We need the following two theorems, which was proved by Sinclair and Tanaka [ST].

Theorem 2.1 ([ST]) Let $M$ be a 2-sphere of revolution with a pair of poles $p, q$ satisfying the following two properties, 
(i) $M$ is symmetric with respect to the reflection fixing $r=a$, where $2 a$ denotes the distance between $p$ and $q$.

(ii) The Gaussian curvature $G$ of $M$ is monotone along a meridian from the point $p$ to the point on $r=a$.

Then the cut locus of a point $x \in M \backslash\{p, q\}$ with $\theta(x)=0$ is a single point or a subarc of the opposite half meridian $\theta=\pi$ (resp. the parallel $r=2 a-r(x)$ ) when $G$ is decreasing (resp. increasing) along a meridian from $p$ to the point on $r=a$. Furthermore, if the cut locus of a point $x \in M \backslash\{p, q\}$ is a single point, then the Gaussian curvature is constant.

Here, we review the notion of a cut point and a cut locus. Let $M$ be a complete Riemannian manifold with a base point $p$. Let $\gamma:[0, a] \rightarrow M$ denote a unit speed minimal geodesic segment emanating from $p=\gamma(0)$ on $M$. If any extended geodesic segment $\bar{\gamma}:[0, b] \rightarrow M$ of $\gamma$, where $b>a$, is not minimizing arc joining $p$ to $\bar{\gamma}(b)$ anymore, then the endpoint $\gamma(a)$ of the geodesic segment is called a cut point of $p$ along $\gamma$. For each point $p$ on $M$, the cut locus $C_{p}$ is defined by the set of all cut points along the minimal geodesic segments emanating from $p$. It is known (for example see [SST] that the cut locus has a local tree structure for 2-dimensional Riemannian manifolds.

Theorem 2.2 Let $M$ be a complete connected $n$-dimensional Riemannian manifold with a base point $p$ such that $M$ has radial sectional curvature at $p$ bounded from below by the radial curvature function of a 2-sphere of revolution $\widetilde{M}$ with a pair of poles $\tilde{p}, \tilde{q}$. Suppose that the cut locus of any point on $\widetilde{M}$ distinct from its two poles is a subset of the half meridian opposite to the point. Then for each geodesic triangle $\triangle(p x y)$ in $M$, there exists a geodesic triangle $\widetilde{\triangle}(p x y):=\triangle(\tilde{p} \tilde{x} \tilde{y})$ in $\widetilde{M}$ such that

$$
d(p, x)=d(\tilde{p}, \tilde{x}), \quad d(p, y)=d(\tilde{p}, \tilde{y}), \quad d(x, y)=d(\tilde{x}, \tilde{y}),
$$

and such that

$$
\angle(p x y) \geqslant \angle(\tilde{p} \tilde{x} \tilde{y}), \quad \angle(p y x) \geqslant \angle(\tilde{p} \tilde{y} \tilde{x}), \quad \angle(x p y) \geqslant \angle(\tilde{x} \tilde{p} \tilde{y}) .
$$

Here, $\angle(p x y)$ denotes the angle at the vertex $x$ of the geodesic triangle $\triangle(p x y)$.

\section{Proof of Main Theorem}

Let $M$ be a complete connected $n$-dimensional Riemannian manifold with a base point $p$ and $\widetilde{M}$ a 2 -sphere of revolution with a pair of poles $\tilde{p}, \tilde{q}$ satisfying (1.1) and (1.2) in the introduction, i.e., a model surface.

From now on, we assume that $M$ has radial sectional curvature at $p$ bounded from below by the radial curvature function of $\widetilde{M}$. By scaling the Riemannian metrics of $M$ and $\widetilde{M}$, we may assume that $2 a=\pi$.

Lemma 3.1 The perimeter of any geodesic triangle $\widetilde{\triangle}($ pxy $)$ of $\widetilde{M}$ does not exceed $2 \pi$, i.e.,

$$
d(\tilde{p}, \tilde{x})+d(\tilde{p}, \tilde{y})+d(\tilde{x}, \tilde{y}) \leqslant 2 \pi .
$$


Proof. Since $d(\tilde{p}, \tilde{q})=2 a=\pi$, it follows from the triangle inequality that

$$
\begin{aligned}
d(\tilde{x}, \tilde{y}) & \leqslant d(\tilde{q}, \tilde{x})+d(\tilde{q}, \tilde{y}) \\
& =(\pi-d(\tilde{p}, \tilde{x}))+(\pi-d(\tilde{p}, \tilde{y})) \\
& =2 \pi-d(\tilde{p}, \tilde{x})-d(\tilde{p}, \tilde{y}) .
\end{aligned}
$$

Therefore, the inequality (3.1) holds.

Lemma 3.2 The perimeter of a geodesic triangle $\triangle($ pxy $)$ of $M$ does not exceed $2 \pi$.

Proof. Let $\triangle(p x y)$ be any geodesic triangle of $M$. From Theorem 2.2, we get a geodesic triangle $\widetilde{\triangle}(p x y)$ of $\widetilde{M}$ satisfying (2.1). Hence, by Lemma 3.1, the perimeter of $\triangle(p x y)$ does not exceed $2 \pi$.

Lemma 3.3 The diameter of $\widetilde{M}$ equals $\pi$, where the diameter diam $\widetilde{M}$ of $\widetilde{M}$ is defined by

$$
\operatorname{diam} \widetilde{M}:=\max \{d(\tilde{x}, \tilde{y}) \mid \tilde{x}, \tilde{y} \in \widetilde{M}\}
$$

Proof. Choose any points $\tilde{x}, \tilde{y}$ on $\widetilde{M}$. By the triangle inequality,

$$
d(\tilde{x}, \tilde{y}) \leqslant d(\tilde{p}, \tilde{x})+d(\tilde{p}, \tilde{y}) .
$$

Thus, by combining (3.1) and (3.2), we obtain

$$
d(\tilde{x}, \tilde{y}) \leqslant \pi=d(\tilde{p}, \tilde{q})
$$

for any $\tilde{x}, \tilde{y}$ on $\widetilde{M}$.

Lemma 3.4 The diameter $\operatorname{diam} M$ of $M$ does not exceed the diameter of $\widetilde{M}$.

Proof. Choose a pair of points $x, y \in M$ satisfying $d(x, y)=\operatorname{diam} M$. Suppose that $x=p$ or $y=p$. By the Rauch comparison theorem, there does not exist a minimal geodesic segment emanating from $p$ whose length exceeds $\pi$, since the manifold $M$ has radial curvature at $\mathrm{p}$ bounded from below by the radial curvature function of the model surface $\widetilde{M}$. Thus, $\operatorname{diam} M=d(x, y) \leqslant \pi$. Suppose that $x \neq p$ and $y \neq p$. Then, for the geodesic triangle $\triangle(p x y)$ in $M$, there exists a triangle geodesic $\widetilde{\triangle}(p x y)$ in $\widetilde{M}$ satisfying (2.1). Hence, we obtain $\operatorname{diam} M=d(\tilde{x}, \tilde{y}) \leqslant \operatorname{diam} \widetilde{M}$.

Lemma 3.5 If $\operatorname{diam} M=\operatorname{diam} \widetilde{M}$, then there exists a point $q \in M$ with $d(p, q)=$ $\operatorname{diam} \widetilde{M}$

Proof. Let $x, y \in M$ be points satisfying $\pi=\operatorname{diam} M=d(x, y)$. Supposing that $x \neq p$ and $y \neq p$, we will get a contradiction. Then, there exists a geodesic triangle $\triangle(p x y)$ with $d(x, y)=\pi$.

It follows from Theorem 2.2 that there exists a geodesic triangle $\widetilde{\triangle}(p x y)$ corresponding to $\triangle(p x y)$ satisfying $d(\tilde{x}, \tilde{y})=d(x, y)=\pi$. By the triangle inequality, $d(\tilde{p}, \tilde{x})+d(\tilde{p}, \tilde{y}) \geqslant$ $d(\tilde{x}, \tilde{y})=\pi$, and Lemma 3.1, we get

$$
d(\tilde{p}, \tilde{x})+d(\tilde{p}, \tilde{y})=\pi=d(\tilde{x}, \tilde{y}) .
$$


This means that the subarc $\alpha$ (passing through $\tilde{p}$ ) of the meridian joining $\tilde{x}$ to $\tilde{y}$ is minimal. Hence the complementary subarc of $\alpha$ in the meridian is also a minimal geodesic segment joining $\tilde{x}$ to $\tilde{y}$, since the length of each meridian is $2 \pi$. Therefore, by Theorem 2.1 $\tilde{y}$ is a unique cut point of $\tilde{x}$ and hence, the Gaussian curvature $G$ of $\widetilde{M}$ is constant. We get a contradiction since $G$ is strictly decreasing along a meridian from $p$ to the point on the equator. This implies the existence of the point $q$.

Lemma 3.6 If there exists a point $q \in M$ with $d(p, q)=\operatorname{diam} \widetilde{M}$, then $q$ is a unique cut point of $p$, and

$$
K(\sigma)=G \circ \mu(d(p, x))
$$

holds for any point $x \in M \backslash\{p\}$ and any radial plane $\sigma$ at $x$.

Proof. Since the point $q$ is a farthest point from $p, q$ is a cut point of $p$. Choose any point $x \in M \backslash\{p, q\}$. By the triangle inequality,

$$
d(p, x)+d(x, q) \geqslant d(p, q)=\pi
$$

and by Lemma 3.2 ,

$$
d(p, x)+d(x, q)+d(p, q) \leqslant 2 \pi
$$

Hence, we get

$$
d(p, x)+d(x, q)=d(p, q)=\pi
$$

and it is easy to see that $q$ is a unique cut point of $p$.

Next, we will prove that $K(\sigma)=G \circ \mu(d(p, x))$ for any $x \in M \backslash\{p, q\}$ and any radial plane $\sigma$ at $x$. Suppose that there exist a point $x \in M \backslash\{p, q\}$ and a radial plane $\sigma$ at $x$ such that $K(\sigma)>G \circ \mu(d(p, x))$. Let $\gamma:[0, \pi] \rightarrow M$ denote the minimal geodesic segment emanating from $p$ passing through $x$. Choose a unit tangent vector $v \in \sigma \subset T_{x} M$ orthogonal to $\gamma^{\prime}(d(p, x))$. Let $Y(t)$ denote the Jacobi field along $\gamma(t)$ satisfying $Y(0)=0$ and $Y(d(p, x))=v$, and hence $\sigma$ is spanned by $Y(d(p, x))$ and $\gamma^{\prime}(d(p, x))$. By the Rauch comparison theorem, there exists a conjugate point $\gamma\left(t_{1}\right)$ of $p$ along $\gamma$ for some $t_{1} \in(0, \pi)$, since $K(\sigma)>G \circ \mu(d(p, x))$ and the sectional curvature of the radial plane spanned by $Y(t)$ and $\gamma^{\prime}(t)$ is not less than $G \circ \mu(t)$ for each $t \in(0, \pi)$. This contradicts the fact that the geodesic segment $\gamma$ is minimal.

Proof of Main Theorem. The first claim is clear from Lemma 3.4. Suppose that $\operatorname{diam} M=\operatorname{diam} \widetilde{M}$. By Lemmas 3.5 and 3.6, $K(\sigma)=G \circ \mu(d(p, x))$ for any point $x \in$ $M \backslash\{p\}$ and any radial plane $\sigma$ at $x$. Thus, it follows from Lemma 1 and Theorem 3 in [KK] that $M$ is isometric to the $n$-dimensional model of $\widetilde{M}$. Incidentally, the explicit isometry $\varphi$ between $M$ and the $n$-dimensional model of $\widetilde{M}$ is given by

$$
\varphi(x):=\left\{\begin{array}{lll}
\exp _{\tilde{p}} \circ I \circ \exp _{p}^{-1}(x) & \text { if } & x \neq q \\
\tilde{q} & \text { if } & x=q,
\end{array}\right.
$$

where $I: T_{p} M \rightarrow T_{\tilde{p}} \widetilde{M}$ denotes a linear isometry and $q$ denotes the unique cut point of $p$. 


\title{
References
}

[Ch] S. Y. Cheng, Eigenvalue comparison theorems and its geometric applications, Math. Z. 143 (1975) 289-297.

[CE] J. Cheeger and D. Ebin, Comparison Theorems in Riemannian Geometry, North-Holland, Amsterdam and New York, 1975.

[KK] Neil N. Katz and Kei Kondo, Generalized space forms, Trans. Amer. Math. Soc. 354 (2002) 2279-2284.

[SST] K. Shiohama, T. Shioya, and M. Tanaka, The Geometry of Total Curvature on Complete Open Surfaces, Cambridge tracts in mathematics 159, Cambridge University Press, Cambridge, 2003.

[ST] R. Sinclair and M. Tanaka, The cut locus of a two-sphere of revolution and Toponogov's comparison theorem, Tohoku Math. J. 59 (2007) 379-399.

\author{
Nathaphon BOONNAM \\ Department of Mathematics \\ Tokai University \\ Hiratsuka City, Kanagawa \\ 259-1292 Japan \\ e-mail : nut4297nb@gmail.com
}

